\title{
Bridging the Synchrotron - Lab Source Gap for Microscopy: The Inverse Compton Scattering X-ray Source
}

\author{
$\underline{\text { M. Feser }}^{1 *}$, R. Ruth ${ }^{1}$, R. Loewen ${ }^{1}$ and J. Kasahara ${ }^{1}$ \\ 1. Lyncean Technologies, Inc. Fremont, CA, USA. \\ * Corresponding author, michael_feser@lynceantech.com
}

Laboratory x-ray sources based on electron impact on a metal target have seen comparatively little improvement since the early days of x-rays. The achievable power and brightness of these sources is limited by target failure. This is caused by the limited thermal conductivity of the metal target material leading to exceeding the melting point of the target locally.

In recent years, new approaches to extend the limits of electron impact sources have been pursued and are being commercialized by companies. Most notably these are sources based on a liquid metal jet commercialized by Excillum [1,2] and structured anode sources commercialized by Sigray [3,4]. In addition to higher power density loading due to embedding microscopic metal structures in a high conductivity material such as diamond, the sources made by Sigray can have different metals on the same target to switch between target materials and use different characteristic lines to generate element specific contrast.

While these improvements in electron impact sources promise an order of magnitude higher brightness and multi-energy capability, there is still a widening performance gap between these sources and what is available at synchrotron sources. Not only do synchrotrons provide many orders of magnitude higher brightness, but they also allow the x-ray energy to be tuned continuously over a wide range making spectroscopic measurements possible that allow for example oxidation state measurements and can give other chemistry or coordination information.

We have been pursuing an alternative approach to bring synchrotron capability to local laboratories based on a miniature synchrotron generating hard x-rays by inverse Compton scattering (ICS) called the Lyncean Compact Light Source (CLS) shown in Figure 1. The miniaturization is accomplished by lowering the energy of the stored electron beam by approximately a factor of 100 compared to regular synchrotrons (From multi $\mathrm{GeV}$ to tens of $\mathrm{MeV}$ ) which allows the shrinking of the circumference of the electron storage ring by the same factor (from hundreds of meters to multiple meters). For the generation of x-rays, the electron beam interacts with a picosecond laser pulse of 1 micrometer wavelength in a frontal collision geometry. The field of the laser pulse acts on the electron beam in the same manner as a magnetic undulator would only at much smaller period. The ICS source is a true miniature synchrotron x-ray source with undulator output x-ray characteristics (inherently monochromatic, tunable, high flux) as shown in Figure 2.

The output beam of the Lyncean CLS is axially symmetric with 4 mrad beam divergence, a $\sim 4 \%$ bandwidth and tunable from 8 to $35 \mathrm{keV}$ by changing the energy of the stored electron beam. It delivers $\mathrm{x}$-rays to experimental end stations located outside of a shielded enclosure that is approximately $8 \mathrm{~m}$ by $10 \mathrm{~m}$ in footprint size and $\sim 80 \mathrm{~cm}$ concrete wall thickness.

The first commercial installation of a Lyncean CLS is at the Technical University Munich (TUM) in Germany [5] which after an upgrade in 2017 delivers now $3 * 10^{10}$ photons/s at 35keV x-ray energy. Specifications of this existing system, the next system under development and future opportunities with additional development are shown in Table 1.

The next commercial system will have a flux and brightness ten times higher than the system in Munich. Most of the performance improvement is due to the use of a new design of the electron storage ring using 
a multi-bend achromat design similar to the design of the latest generation synchrotron storage rings such as Max IV.

Applications pursued with the Munich CLS are primarily Talbot grating based multi-modal imaging and tomography (quantitative absorption/phase contrast, dark field) and high-resolution x-ray tomography. The Lyncean CLS is very well matched to the needs of these measurements due to the inherent coherence property, Monochromaticity, which avoids beam hardening and enhances quantitation, and the high flux. Analytical applications using specifically developed multilayer focusing optics have been demonstrated at the Lyncean factory in the USA. Protein crystallography with freely selectable x-ray energy to enable advanced phasing techniques such as single wavelength anomalous dispersion (SAD) are possible. Other examples include powder diffraction and small angle scattering, to name a few.

The special characteristic of the Lyncean CLS of producing a truly symmetric and monochromatic beam without contamination by higher $\mathrm{x}$-ray energies (compared to traditional synchrotrons) allows very simple beam transport systems and experimental stations with relaxed shielding requirements to be utilized.

The Lyncean Compact Light Source provides an opportunity to conduct measurements that typically would be relegated to large synchrotron bending magnet sources in a compact form factor. Due to its limited spatial and temporal coherence, applications that require a fully coherent beam such as Ptychography will still be limited to synchrotron undulator beamlines.

\section{References}

[1] M. Otendal, et al, Review of Scientific Instruments 79(1) (2008), p. 016102.

[2] http://www.excillum.com

[3] W. Yun, et al, Microscopy and Microanalysis 22(Suppl 3) (2016), p. 118.

[4] http://www.sigray.com

[5] E. Eggl, et al, Journal of synchrotron radiation 23(5) (2016), p. 1137-1142.

\begin{tabular}{|c|c|c|c|}
\hline Parameter & $\begin{array}{l}\text { CLS } 1.1 \\
\text { (Munich CLS) }\end{array}$ & $\begin{array}{l}\text { CLS } 2.0 \\
\text { (Next system) }\end{array}$ & $\begin{array}{l}\text { CLS Roadmap } \\
\text { (Future) }\end{array}$ \\
\hline Total Flux ( 4\% BW) $-[\mathrm{ph} / \mathrm{s}]$ & $>3 * 10^{10}$ & $4 * 10^{11}$ & $4 * 10^{12}$ \\
\hline Source size $[\mu \mathrm{m} \mathrm{rms}]$ & 45 & 40 & 30 \\
\hline $\begin{array}{l}\text { Source divergence [mrad] } \\
\text { (Flattop Cone) }\end{array}$ & 4 & 6 & 6 \\
\hline $\begin{array}{l}\text { Source Brightness - Full Bandwidth } \\
{\left[\mathrm{ph} / \mathrm{s} / \mathrm{mrad}^{\wedge} 2 / \mathrm{mm}^{\wedge} 2 / 4 \% \mathrm{BW}\right]}\end{array}$ & $5 * 10^{11}$ & $4 * 10^{12}$ & $1 * 10^{14}$ \\
\hline Tunable x-ray energy range [keV] & $\begin{array}{l}\text { 8-35 } \\
\text { (IR 1um laser) }\end{array}$ & $\begin{array}{l}\text { 8-35 } \\
\text { (IR 1um laser) }\end{array}$ & $\begin{array}{l}4-22 \text { (IR }-2 \text { um) } \\
8-50 \text { (IR }-1 \text { um) } \\
16-100 \text { (Vis }-0.5 \text { um) }\end{array}$ \\
\hline X-ray energy bandwidth [dE/E FWHM] & $3-5 \%$ & $3-5 \%$ & $2-4 \%$ \\
\hline X-ray Pulse Length (rms) [ps] & 60 & & \\
\hline X-ray Repetition Rate [MHz] & 65 & & \\
\hline
\end{tabular}

Table1: Performance specifications of the system installed in Munich, the next production system that will be built, and the target parameters that can be achieved in the future with additional development. 


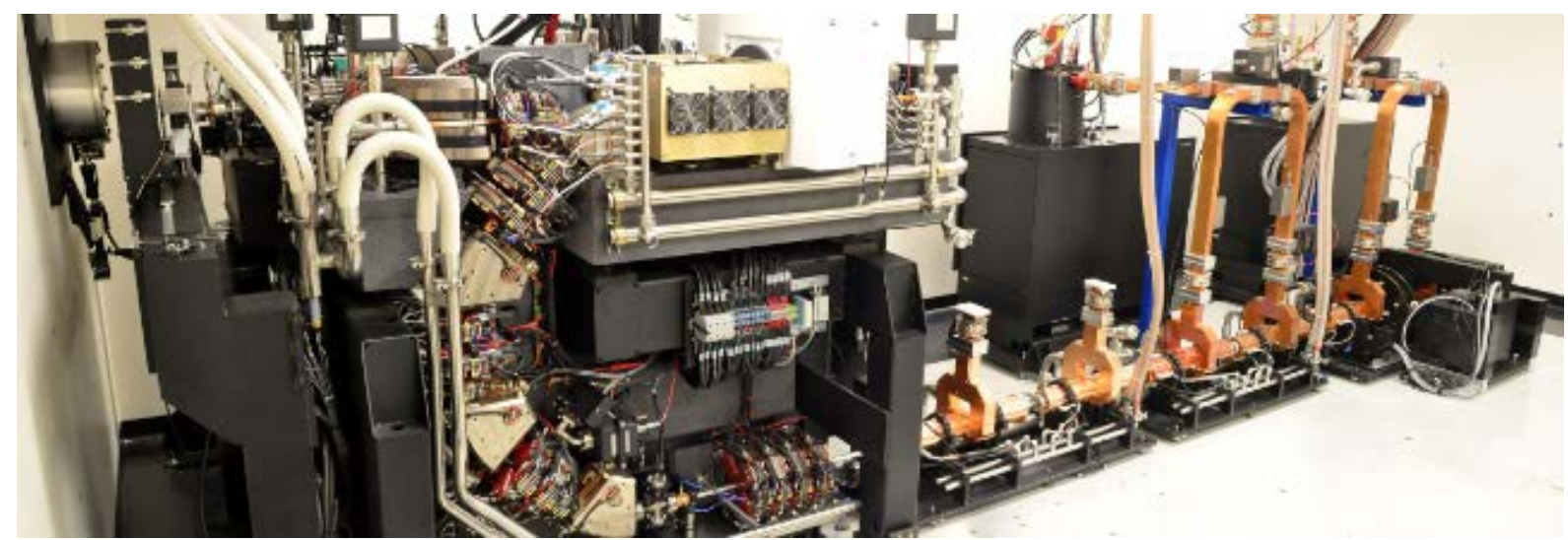

Figure 1: Photograph of the Lyncean Compact Light Source. The dimensions of the source are approximately $8 \mathrm{~m}$ x $4 \mathrm{~m}$.
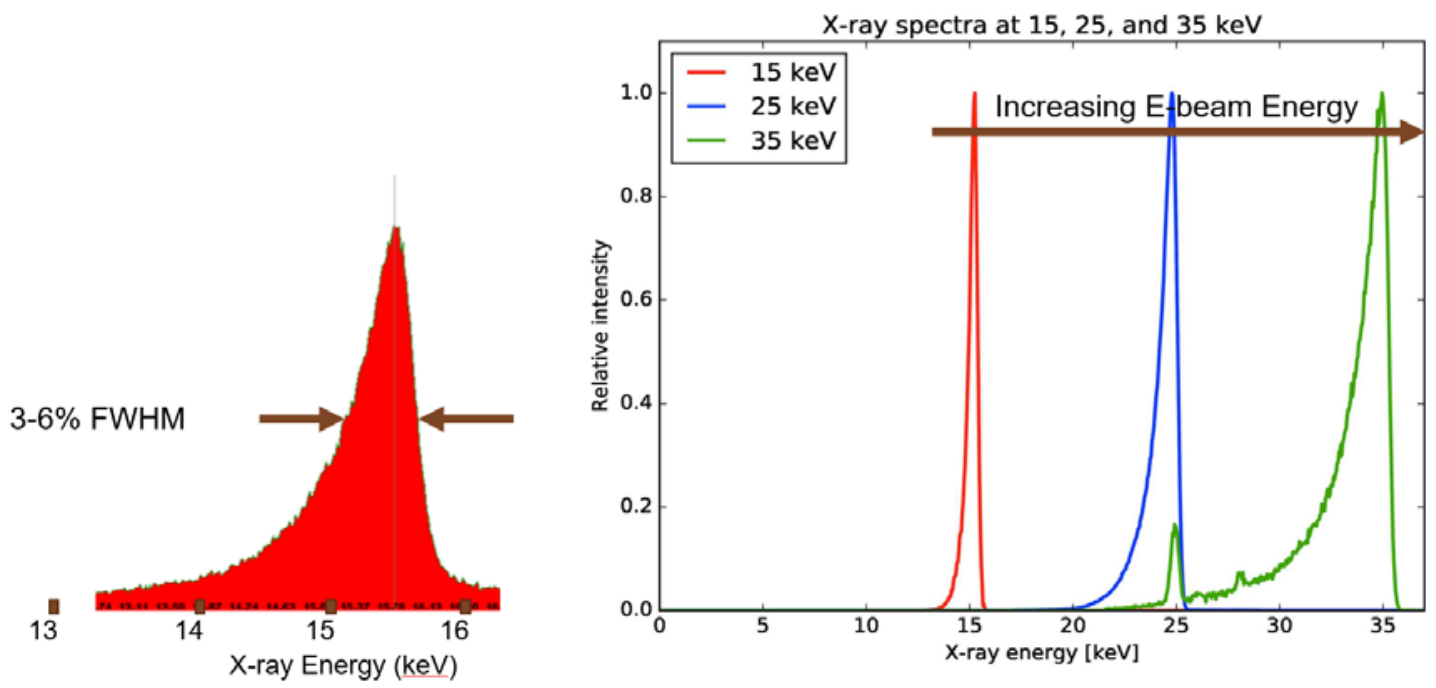

Figure 2: Measured output spectra of the Lyncean CLS at different electron energy configurations. Depending on the electron energy the energy spread is in the range of 3-6\% FWHM (lowest at highest energy). The $\mathrm{x}$-ray energy is continuously tunable by changing the energy of the stored electrons in the storage ring. 
https://doi.org/10.1017/S1431927618013879 Published online by Cambridge University Press 